EPJ Web of Conferences 59, 15001 (2013)

DOI: $10.1051 /$ epjconf/20135915001

(C) Owned by the authors, published by EDP Sciences, 2013

\title{
High-power laser experiments to study collisionless shock generation
}

\author{
Y. Sakawa ${ }^{1, a}$, Y. Kuramitsu ${ }^{1}$, T. Morita ${ }^{1}$, T. Kato ${ }^{2}$, H. Tanji ${ }^{3}$, T. Ide ${ }^{3}$, K. Nishio ${ }^{4}$, \\ M. Kuwada ${ }^{4}$, T. Tsubouchi ${ }^{3}$, H. Ide ${ }^{4}$, T. Norimatsu ${ }^{1}$, C. Gregory ${ }^{5}$, N. Woolsey ${ }^{5}$, \\ K. Schaar ${ }^{6}$, C. Murphy ${ }^{6}$, G. Gregori ${ }^{6}$, A. Diziere ${ }^{7}$, A. Pelka ${ }^{7}$, M. Koenig ${ }^{7}$, \\ S. Wang ${ }^{8}$, Q. Dong ${ }^{8}$, Y. Li ${ }^{8}$, H.-S. Park ${ }^{9}$, S. Ross ${ }^{9}$, N. Kugland ${ }^{9}$, D. Ryutov ${ }^{9}$, \\ B. Remington ${ }^{9}$, A. Spitkovsky ${ }^{10}$, D. Froula ${ }^{11}$ and H. Takabe ${ }^{1}$
}

1 Institute of Laser Engineering, Osaka University 2-6, Yamadaoka, Suita 565-0871, Japan

2 Graduate School of Science, Hiroshima Univ., 1-3-1, Kagamiyama, Higashi-Hiroshima 739-8526, Japan

3 Graduate School of Engineering, Osaka University 2-1, Yamadaoka, Suita 565-0871, Japan

4 Graduate School of Science, Osaka University 1-1, Machikaneyama, Toyonaka 560-0043,

Japan

${ }^{5}$ Department of Physics, University of York, Heslington YO105DD, UK

${ }^{6}$ Department of Physics, Oxford University, Oxford OX1 3PU, UK

7 LULI Ecole Polytechnique, 91128 Palaiseau Cedex, France

8 Institute of Physics, Chinese Academy of Sciences, Beijing 100190, China

${ }^{9}$ Lawrence Livermore National Lab, 7000 East Ave, Livermore, CA 94550, USA

10 Department of Astrophysical Sciences, Princeton University, Princeton, NJ 08544, USA

11 Laboratory for Laser Energetics, 250 East River Road, Rochester, NY 14623, USA

\begin{abstract}
A collisionless Weibel-instability mediated shock in a self-generated magnetic field is studied using two-dimensional particle-in-cell simulation [Kato and Takabe, Astophys. J. Lett. 681, L93 (2008)]. It is predicted that the generation of the Weibel shock requires to use NIF-class high-power laser system. Collisionless electrostatic shocks are produced in counter-streaming plasmas using Gekko XII laser system [Kuramitsu et al., Phys. Rev. Lett. 106, 175002 (2011)]. A NIF facility time proposal is approved to study the formation of the collisionless Weibel shock. OMEGA and OMEGA EP experiments have been started to study the plasma conditions of counter-streaming plasmas required for the NIF experiment using Thomson scattering and to develop proton radiography diagnostics.
\end{abstract}

\section{INTRODUCTION}

Collisionless shocks are observed in astrophysical plasmas. For example in a shock wave observed in a supernova remnant, a Coulomb mean-free-path is much longer than the shock-front thickness. Large amplitude turbulent waves and energetic particles are also observed in the shock environments. Diffusive shock acceleration is considered to be a standard model for non-thermal acceleration of energetic particles or cosmic rays in the universe. On the other hand, in astrophysical plasmas, there is no way to directly measure the key quantities to investigate the shock dynamics and the particle acceleration. One

\footnotetext{
ae-mail: sakawa-y@ile.osaka-u.ac.jp
}

This is an Open Access article distributed under the terms of the Creative Commons Attribution License 2.0, which permits unrestricted use, distribution, and reproduction in any medium, provided the original work is properly cited. 


\section{EPJ Web of Conferences}

can observe the X-ray emission from the vicinity of the shock-front; however, there are significant uncertainties in the physics surrounding particle acceleration by collisionless shocks. A laboratory experiment can be an alternative approach to study collisionless shocks and particle acceleration.

In this paper, we investigate results of particle-in-cell (PIC) simulation and laboratory experiments using high-power laser systems to study collisionless shock generation in counter-streaming plasmas.

\section{NUMERICAL STUDIES}

Recent numerical and PIC simulation studies show that there are two possible collisionless shocks in unmagnetized plasmas: One is an electrostatic (ES) shock [1, 2], and the other is a Weibel-instability mediated shock in self-generated magnetic field [3,4].

Kato and Takabe investigated the collisionless Weibel shock in two-dimensional (2D) PIC simulation using the injection method [3]. Both electrons and ions are located in the region between the two rigid walls at the left- and right-sides of the simulation box with the bulk flow-velocity of $\mathrm{V}$ in the $+\mathrm{x}$ direction (towards the right-wall) and reflected at the right-wall. Figure 1(a) shows the ion density normalized by the upstream (left-side) electron density $\mathrm{n}_{\mathrm{i}} / \mathrm{n}_{\mathrm{e} 0}$ at $\omega_{\mathrm{pe}} \mathrm{t}=2100$ for $\mathrm{V}=0.45 \mathrm{c}$ ( $\mathrm{c}$ is the speed of light) and ion-to-electron mass ratio of $\mathrm{m}_{\mathrm{i}} / \mathrm{m}_{\mathrm{e}}=20$. Here, we take $\omega_{\mathrm{pe}}^{-1}\left(\omega_{\mathrm{pe}}\right.$ is the electron plasma frequency) as the unit of time and the electron skin depth $\lambda_{\mathrm{e}}=\mathrm{c} \omega_{\mathrm{pe}}^{-1}$ as the unit of length. The upstream plasma flows from the left to the right and goes through the transition region, which has a filamentary structure, and then reaches the almost uniform downstream state at $\mathrm{x} / \lambda_{\mathrm{e}}>1900$. Figure 1(b) shows the time evolution of $n_{i} / n_{e 0}$. The transition region or shock front is visible as a steep increase in the ion density after $\omega_{\mathrm{pe}} \mathrm{t} \sim 500$. Figures $1(\mathrm{c})$ and $1(\mathrm{~d})$ represent $\mathrm{V}$ dependence of $\mathrm{x}$-profiles of $\mathrm{n}_{\mathrm{i}} / \mathrm{n}_{\mathrm{e} 0}$ and the energy densities of magnetic fields normalized by the upstream bulk kinetic-energy density $\mathrm{U}_{\mathrm{B}} / \mathrm{U}_{\mathrm{KE}}$, respectively. Here, $\mathrm{U}_{\mathrm{KE}}=\mathrm{n}_{\mathrm{e} 0}\left(\mathrm{~m}_{\mathrm{i}}+\mathrm{m}_{\mathrm{e}}\right) \mathrm{V}^{2} / 2$. As the unit of length we define the ion inertial length $\lambda_{\mathrm{i}}=\left(\mathrm{m}_{\mathrm{i}} / \mathrm{m}_{\mathrm{e}}\right)^{0.5} \lambda_{\mathrm{e}}$ in Figs. 1(c) and 1(d). We see that $\mathrm{n}_{\mathrm{i}} / \mathrm{n}_{\mathrm{e} 0}$ [Fig. 1(c)] profiles are almost independent of $\mathrm{V}$, and $\mathrm{U}_{\mathrm{B}} / \mathrm{U}_{\mathrm{KE}}$ [Fig. 1(d)] profiles show small variation with $\mathrm{V}$. The widths of the shock transition region are $\mathrm{W}-100 \lambda_{\mathrm{i}}$ in all cases. A strong magnetic field is generated at the shock transition region, and $\mathrm{U}_{\mathrm{B}} / \mathrm{U}_{\mathrm{KE}}$ reaches $1-2 \%$. This strong magnetic field provides an effective dissipation mechanism for this collisionless shock.

A scaling-law derived by changing $\mathrm{V} / \mathrm{c}(=0.1,0.2$ and 0.45$)$ and $\mathrm{m}_{\mathrm{i}} / \mathrm{m}_{\mathrm{e}}(=20,50,100)$ in simulation revealed that high-density (electron density $\left.\sim 10^{20} \mathrm{~cm}^{-3}\right)$, high-flow velocity $(\sim 1000 \mathrm{~km} / \mathrm{s}$ ), and large volume (plasma length $\sim 30 \mathrm{~mm}$ ) $\mathrm{CH}$ plasmas (average mass number $\mathrm{A}=7.5$ and charge $\mathrm{Z}=3.5$ ) are required to produce the collisionless Weibel shock. In order to achieve these plasma parameters, NIF class high-power laser system is required. Using kJ-class laser system, only the collisionless ES shocks can be generated.

\section{EXPERIMENT}

Under international collaborations, we have performed several series of experiments on the high-mach number collisionless ES shock formations using Shenguang II laser system in China [5] and Gekkko XII HIPER laser system in Japan [6].

In Shenguang II experiment, in order to produce collisionless counter-streaming plasmas, a plastic $(\mathrm{CH})$ double-plane target $(100 \mu \mathrm{m}$ in thickness and $4.5 \mathrm{~mm}$ in separation) was used, and 4 beams $[352 \mathrm{~nm}(3 \omega), 1 \mathrm{~ns}, \sim 260 \mathrm{~J} /$ beam $\times 4$ beams, $150 \mu \mathrm{m}$ in spot diameter, laser intensity $\left.\sim 6 \times 10^{15} \mathrm{~W} / \mathrm{cm}^{2}\right]$ were focused on the inner-surface of the $1 \mathrm{st} \mathrm{CH}$ plane. A laser-ablated plasma is formed at the $1 \mathrm{st} \mathrm{CH}$ plane, and the $2 \mathrm{nd} \mathrm{CH}$ plasma is created by radiation and the plasma from the $1 \mathrm{st}$ $\mathrm{CH}$ plane. The interferograms and shadowgraphs were taken transverse to the main laser propagation direction using a probe laser [Nd: YAG laser, $527 \mathrm{~nm}(2 \omega), 70 \mathrm{ps}$ ] with ICCD cameras. As a result, a 

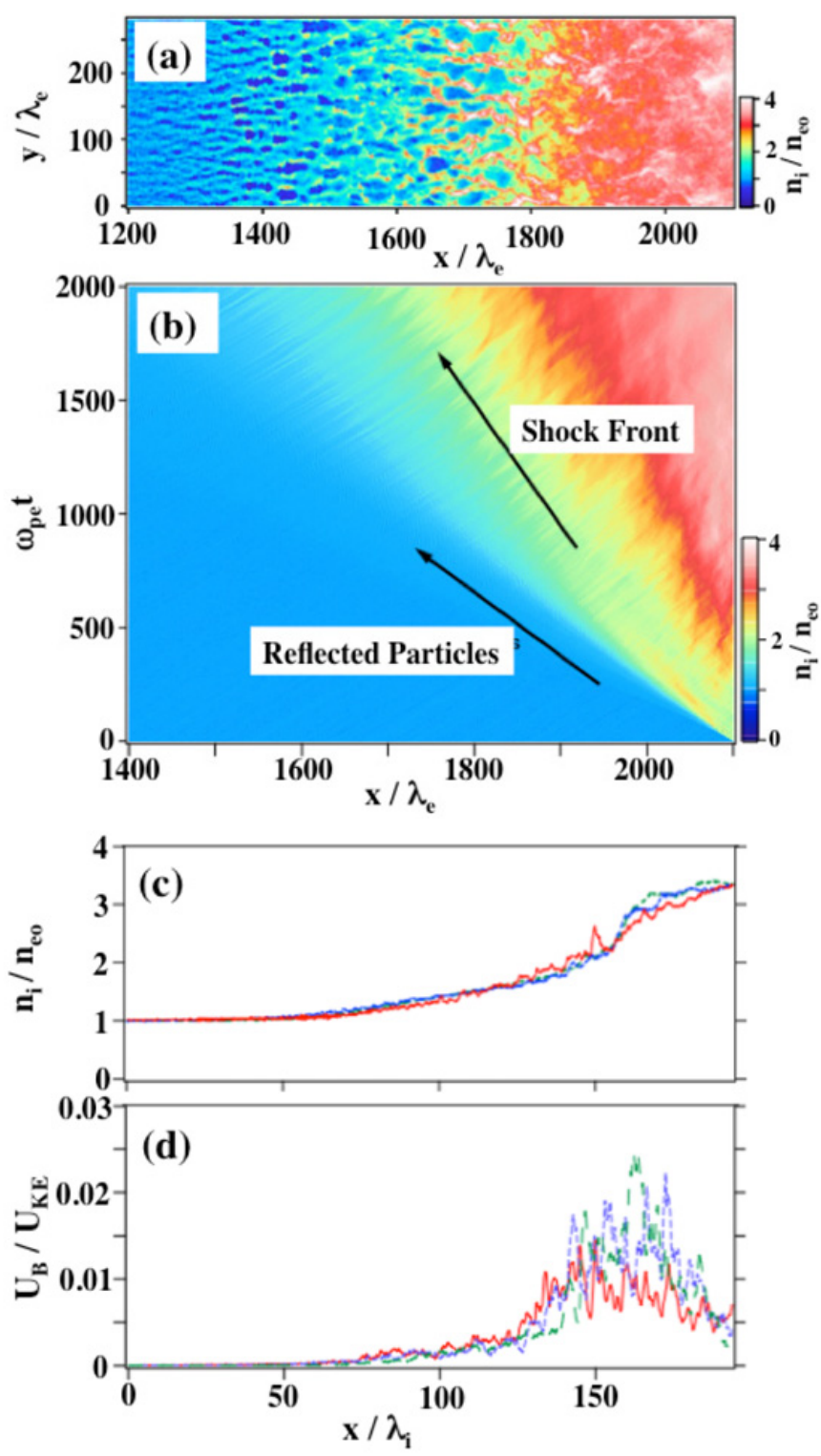

Figure 1. (a) Ion density profile at $\omega_{\mathrm{pe}} \mathrm{t}=2100$. (b) Time evolution of the ion density. The ion density is averaged over the y-direction. Profiles of (c) $\mathrm{n}_{\mathrm{i}} / \mathrm{n}_{\mathrm{e} 0}$ and (d) $\mathrm{U}_{\mathrm{B}} / \mathrm{U}_{\mathrm{KE}}$ for $\mathrm{V}=0.45 \mathrm{c}$ (red solid curves), $0.2 \mathrm{c}$ (blue short-dashed curves), and $0.1 \mathrm{c}$ (green long - dashed curves) [3].

large density-jump with downstream- to upstream-density ratio of $\mathrm{n}_{1} / \mathrm{n}_{0} \sim 3.9$ was observed at an ES shock, indicating a high Mach-number shock [5].

In Gekko XII experiment [ $352 \mathrm{~nm}(3 \omega), 500 \mathrm{ps}, \sim 100 \mathrm{~J}, 300 \mu \mathrm{m}$ in spot diameter, laser intensity $\left.\sim 3 \times 10^{14} \mathrm{~W} / \mathrm{cm}^{2}\right]$, a CH double-plane target $(60 \mu \mathrm{m}$ in thickness and $4.5 \mathrm{~mm}$ in separation) was irradiated by a laser beam onto the inner-surface of the 1st $\mathrm{CH}$ plane as shown in Fig. 2(a) [6]. The plasmas and shocks were diagnosed transverse to the main laser propagation direction; shadowgraphy and interferometry using a probe laser [Nd: YAG laser, $527 \mathrm{~nm}(2 \omega), \sim 10 \mathrm{~ns}]$ with ICCD and 

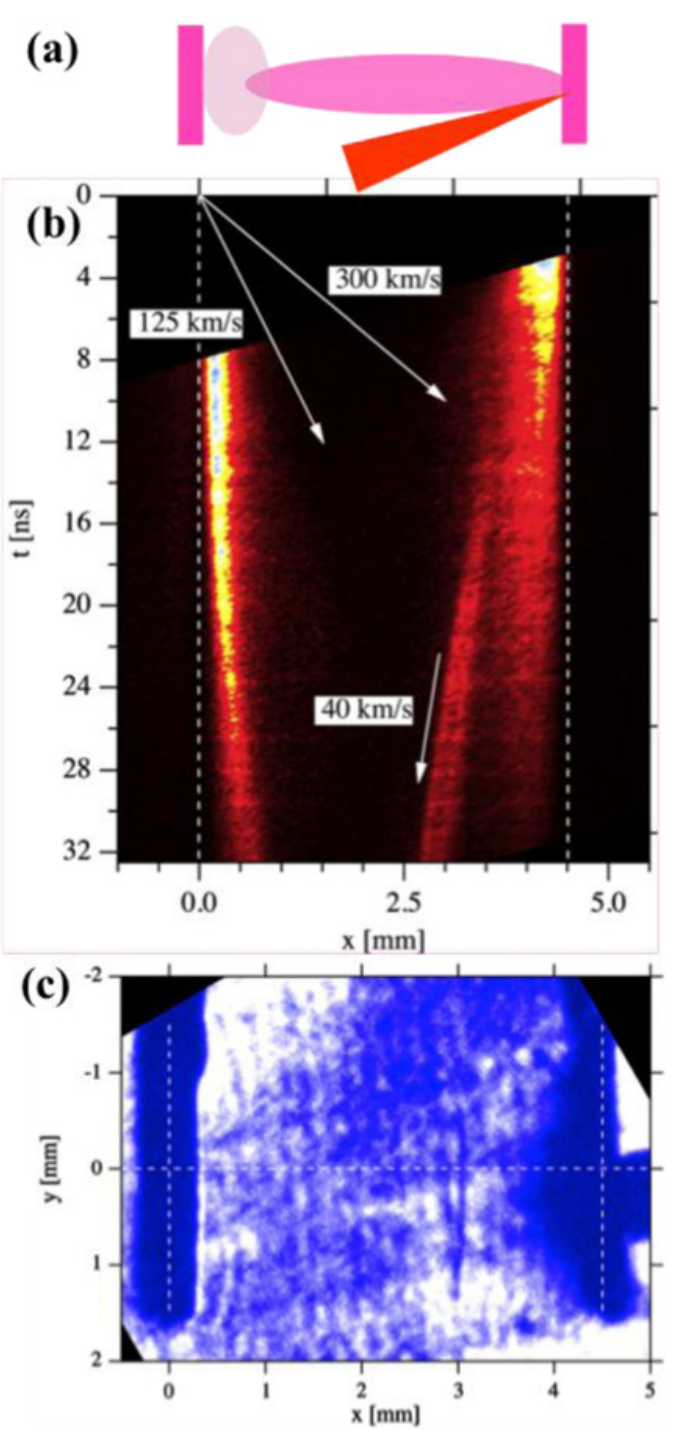

Figure 2. (a) Schematic of the double-plane target. Laser axis is $30^{\circ}$ from the target normal. (b) SOP image. (c) Shadowgraphy snapshot at $\mathrm{t}=25 \mathrm{~ns}[6]$.

streak cameras, and visible $(450 \mathrm{~nm})$ self-emission measurements with ICCD and streak cameras. Figure 2(b) shows streaked self-emission optical pyrometer (SOP) image. Counter-streaming plasmas were produced, and we successfully observed time evolution of an ES shock structure, which is clear at $x \sim 3 \mathrm{~mm}$ by shadowgraphy [Fig. 2(c)] and self-emission measurement (not shown) [6].

In order to demonstrate the formation of collisionless shocks through the self-generated magnetic fields due to the nonlinearity in the growth of the Weibel instability, we have applied to the NIF facility time proposal 2010, "Collisionless shock generation mediated by Weibel instability in counter-streaming ablation plasmas by NIF" (PI: Y. Sakawa). It was approved as a combined experiment with "Laboratory simulation of cosmological magnetic fields and cosmic ray generation (PI: G. Gregori) as a threeyear experiment, and the experiment will be conducted from $\sim 2013$ when the required diagnostics, for example optical interferometry, are constructed. 
We started OMEGA and OMEGA EP experiments [PI: H.-S. Park (LLNL) and PI: A. Spitkovski (Princeton Univ.)] to study the plasma parameters of counter-streaming plasmas required for the NIF experiment using Thomson scattering, and to develop proton-radiography diagnostics for the current filaments produced by the Weibel instability, self-generated magnetic field, and shock-structure measurements. In the OMEGA experiment, 10 laser beams $\left(1 \mathrm{~ns}, 500 \mathrm{~J} /\right.$ beam, $\left.\sim 10^{16} \mathrm{~W} / \mathrm{cm}^{2}\right)$ were focused on each plane of $\mathrm{CH}$ double-plane target with 8-mm separation. It was demonstrated using Thomson scattering measurements that a plasma with an electron density $\sim 10^{19} \mathrm{~cm}^{-3}$ and flow velocity $\sim 1000 \mathrm{~km} / \mathrm{s}$ was created at $4 \mathrm{~mm}$ from the target (middle of the two $\mathrm{CH}$ planes) at $\sim 5 \mathrm{~ns}$ form the laser timing. Proton radiography using OMEGA EP laser with two long-pulse beams for counter-streaming plasma production $[352 \mathrm{~nm}(3 \omega, 3 \mathrm{~ns}, \sim 2.2 \mathrm{~kJ} /$ beam $)$ and two short-pulse beams for two-channel proton radiography $[1.05 \mu \mathrm{m}(\omega), 10 \mathrm{ps}, 250 \mathrm{~J} / \mathrm{beam}]$ showed interesting filamentary and shock-like structures.

\section{SUMMARY}

The collisionless Weibel-instability mediated shock in self-generated magnetic field is studied using 2D PIC simulation, and it is predicted that the generation of the Weibel shock requires NIF class high-power laser system. Collisionless ES shocks are produced in counter-streaming plasmas using lower-power laser systems: Shenguang II [5] and Gekko XII [6]. A NIF facility time proposal is approved to study the formation of the collisionless Weibel skock as a three-year experiment. OMEGA and OMEGA EP experiments have started to study the plasma conditions of counter-streaming plasmas using Thomson scattering and to develop proton-radiography diagnostics.

A part of the experiments was performed under the joint-research project of the Institute of Laser Engineering, Osaka University, and supported by Grant-in-Aid for Scientific Research (B), MEXT of Japan (No. 21340172), and by a grant for the Core-to-Core Program from the Japan Society for the Promotion of Science (JSPS).

\section{References}

[1] G. Sorasio et al., Phys. Rev. Lett. 96, 045005 (2006)

[2] T. N. Kato and H. Takabe, Phys. Plasmas 17, 032114 (2010)

[3] T. N. Kato and H. Takabe, The Astophys. J. Lett. 681, L93 (2008)

[4] H. Takabe et al., Plasma Phys. Controlled Fusion 50, 124057 (2008)

[5] T. Morita et al., Phys. Plasmas 17, 122702 (2010)

[6] Y. Kuramitsu et al., Phys. Rev. Lett. 106, 175002 (2011) 